Guo-Hao Lin

Glendale Lim

Hsun-Liang Chan

William V. Giannobile

Hom-Lay Wang
Review

\section{Recombinant human bone morphogenetic protein 2 outcomes for maxillary sinus floor augmentation: a systematic review and meta-analysis}

Key words: bone substitutes, growth factors, morphometric analysis, sinus floor elevation, wound healing

Guo-Hao Lin, Glendale Lim, Hsun-Liang Chan, William V. Giannobile, Hom-Lay Wang, Graduate

Periodontics, Department of Periodontics \& Oral

Medicine, University of Michigan School of

Dentistry, Ann Arbor, MI, USA

William V. Giannobile, Department of Biomedical Engineering, College of Engineering, University of Michigan, Ann Arbor, MI, USA

\section{Corresponding author:}

Dr. Guo-Hao Lin

Room 1324, 1011 North University Avenue

Ann Arbor, MI 48109-1078, USA

Tel.: (734) 7633325

Fax: (734) 9360374

e-mail: ghlin@umich.edu

Date:

Accepted 8 October 2015

To cite this article:

Lin G-H, Lim G, Chan H-L, Giannobile WV, Wang H-L.

Recombinant human bone morphogenetic protein 2 outcomes

for maxillary sinus floor augmentation: a systematic review

and meta-analysis.

Clin. Oral Impl. Res. 27, 2016, 1349-1359

doi: $10.1111 /$ clr. 12737

\section{Abstract}

Aims: To study the effect of the recombinant human bone morphogenetic protein 2 (rhBMP-2) on sinus volumetric and histometric changes after sinus floor augmentation compared to a conventional approach of non-biologic bone grafting materials.

Materials and methods: An electronic search of 4 databases (January 1990-February 2015), including PubMed/MEDLINE, EMBASE, Web of Science and Cochrane Central, and a hand search of peer-reviewed journals for relevant articles were performed. Human clinical trials with data on comparison of sinus volumetric and/or histometric outcomes with and without the use of rhBMP-2 in sinus grafting procedures, with $\geq 10$ augmentation sites in each study group, and with a followup period of at least 6 months, were included. Random-effects meta-analyses were performed to analyze weighted mean difference (WMD) and confidence interval $(\mathrm{Cl})$ for the recorded variables according to PRISMA guidelines.

Results: Six randomized controlled trials (RCTs) were included. The results of the meta-analyses showed that the WMD of vertical bone height gain was $-0.14 \mathrm{~mm}(95 \% \mathrm{Cl}=-1.91$ to $1.62 \mathrm{~mm}$, $P=0.87)$, the WMD of bone density was $-142.42 \mathrm{mg} / \mathrm{cm}^{3}\left(95 \% \mathrm{Cl}=-310.62-25.78 \mathrm{mg} / \mathrm{cm}^{3}\right.$, $P=0.10)$, the WMD of the percentage of vital bone was $-4.59 \%(95 \% \mathrm{Cl}=-11.73-2.56 \%$, $P=0.21)$, and the WMD of the percentage of residual bone grafting materials was $-9.90 \%(95 \%$ $\mathrm{Cl}=-26.38-6.58 \%, P=0.21)$. The comparison of implant survival rate presented an overall risk ratio of $1.00(95 \% \mathrm{Cl}=0.94-1.07)$. The two approaches (conventional bone grafting compared to BMPs) demonstrated comparable effectiveness for both clinical and histomorphometric measures. Conclusions: This systematic review revealed that the use of rhBMP-2 in maxillary sinus floor augmentation achieved similar clinical and histometric outcomes when compared to conventional sinus grafting procedures after a healing period of 6-9 months. However, previous studies showed the morbidity and other patient-reported outcomes were improved in rhBMP-2 approaches as compared to bone autograft procedures (both intraoral and extraoral bone harvesting because no donor site is required). Long-term studies are required to determine the cost-benefit of sinus floor augmentation procedures for patients requiring implant reconstruction.

\section{Introduction}

Bone formation, maintenance, and regeneration involve a cascade of complex cellular interactions in the signaling pathway. Bone morphogenetic proteins (BMPs) are a group of naturally formed multifunctional growth actors found in human body and are part of the transforming growth factor $\beta$ (TGF- $\beta$ ) superfamily. BMPs initiate the signaling pathway by binding to specific serine-threonine kinase cell surface receptors that phosphorylate and activate the downstream cascade of intracellular proteins interactions. Ultimately, the intracellular proteins promote gene expression by forming several regulatory complexes and bind to the promoter regions of target genes in the nucleus.

BMPs, including BMP-2, are involved in several critical pathways that influence osteoblastogenesis and bone formation: Smad pathway, Hedgehog pathway, TGF- $\beta$ pathway, and cytokine-cytokine receptor interaction (Fig. 1). BMP-2 target genes include a wide cohort of transcription factors located in cell nucleus. The osteogenic effects of 
BMP-2 are mediated by formation of Runx2Smad complexes (Hassan et al. 2006). Smads, the TGF- $\beta$ intracellular proteins, form regulatory complexes with the transcription factors to regulate the downstream phenotypic target genes induction. BMP-2 promotes the osteoblast maturation by increasing the upregulation expression of series of transcription factors in cell nucleus (RUNX2, OSX, DLX5), which then lead to the expression of OSE2, the osteoblast marker gene that is responsible for the osteoblast differentiation (Komori et al. 1997).

BMPs activity was first identified when demineralized bone matrix-induced preosteoprogenitor cells to differentiate into osteoblasts and facilitate new bone formation when implanted in extraskeletal sites (Urist 1965). The biologic and cellular functions of BMPs were later discovered through in vitro and in vivo studies. In addition to ectopic bone and cartilage development and regeneration process (Sporn \& Vilcek 1996), BMPs are also involved in numbers of nonosteogenic development process: BMPs play critical roles in heart and neural development. This group of growth factors are involved in the differentiation of epidermal, adrenergic phenotype in developing neurons, chondrocyte, and osteoblast precursors (Chen et al. 2004).

Since the discovery of BMP's osteoinductive ability, much of the research focus has been on the therapeutic application of specific BMP isoforms in regenerative therapy. In recent preclinical and human studies, the use of BMP-2, 4, 6, 7, 9, and 14 showed promising osteoinduction in repairing radial, femoral, spinal fusion, and cranial bone defects (Sigurdsson et al. 1997; Govender et al. 2002; Dai et al. 2005; Stavropoulos et al. 2011; Even et al. 2012; Carreira et al. 2014). Based on radiographic, histological, and mechanical evaluation, the osseous defects resulted in successful healing of segmental defects. In sight of the commonality of dentoalveolar defects, Bowers integrated BMP-2 into periodontal grafting material and successfully regenerated significant amount of periodontal attachment apparatus including new cementum, connective tissue, and new bone (Bowers et al. 1991). Some studies had also shown successful application of BMP-2 in repairing peri-implant defects and induce implant osteointegration (Sigurdsson et al. 1997). However, the use of BMPs in clinical setting tends to be restricted due to the technique sensitive protein extraction procedure and low product yield. With the aid of DNA recombinant bioengineering technology, more than 20 types of BMPs have been cloned and characterized (Even et al. 2012). Further, recombinant human available for therapeutic use given its rapid ability to trigger the differentiation of osteoblasts.

Loss of crestal alveolar bone and increased maxillary sinus pneumatization are common clinical sequelae afflicting the edentulous posterior maxilla (Sharan \& Madjar 2008). Surgical approaches such as total or segmental bone onlays, interpositional bone graft, and grafting of the maxillary sinus were developed to incorporate the use of different types of bone graft material for sinus floor augmentation (Chiapasco et al. 2009). Although autogenous bone has been considered as the gold standard for augmentation procedures based on the high success rate (Esposito et al. 2009), it has limited availarea as donor site. Studies with animal models have demonstrated the high osteogenic activity of rhBMP-2; rhBMP-2 seemed to produce similar bone apposition results as autogenous bone (Wada et al. 2001; Lee et al. 2013). Additionally, a human study further demonstrated that the administration of rhBMP-2 via impregnated on absorbable collagen sponge (ACS) in a 2-stage maxillary sinus floor procedure induced de novo bone growth (Boyne et al. 1997). In the study, new bone growth was observed in $100 \%$ of the 11 evaluated patients with BMP-2 (rhBMP-2) has been made readily ability and may require another surgical

histology evidence of normal remodeling and maturation activity in the rhBMP-2induced bone. However, to further warrant the clinical and histometric outcomes of introducing rhBMP-2 in maxillary sinus floor augmentation procedures, a critical systematic review will be needed. Hence, this study aims to investigate the effect of the rhBMP-2 on sinus volumetric and histometric changes after sinus floor augmentation compared with the conventional approach, which is the use of bone grafting materials without additional biologics or growth factors.

\section{Materials and methods}

\section{Patient, intervention, comparison, outcome (PICO) question}

P: Healthy patients receiving sinus floor augmentation procedures.

I: Human clinical trials with data on sinus volumetric and/or histometric outcomes in sinus grafting procedures, with $\geq 10$ augmentation sites in each study group, and with a follow-up period of at least 6 months.

C: The effect of rhBMP-2 on sinus volumetric and histometric changes after sinus floor augmentation compared with the use of bone grafting materials without an addition of biologic agents.

O: Risk ratio of implant survival rates (SR) and weighted mean difference (WMD) of vertical bone level (VBL) gain, bone density, and histometric outcomes.

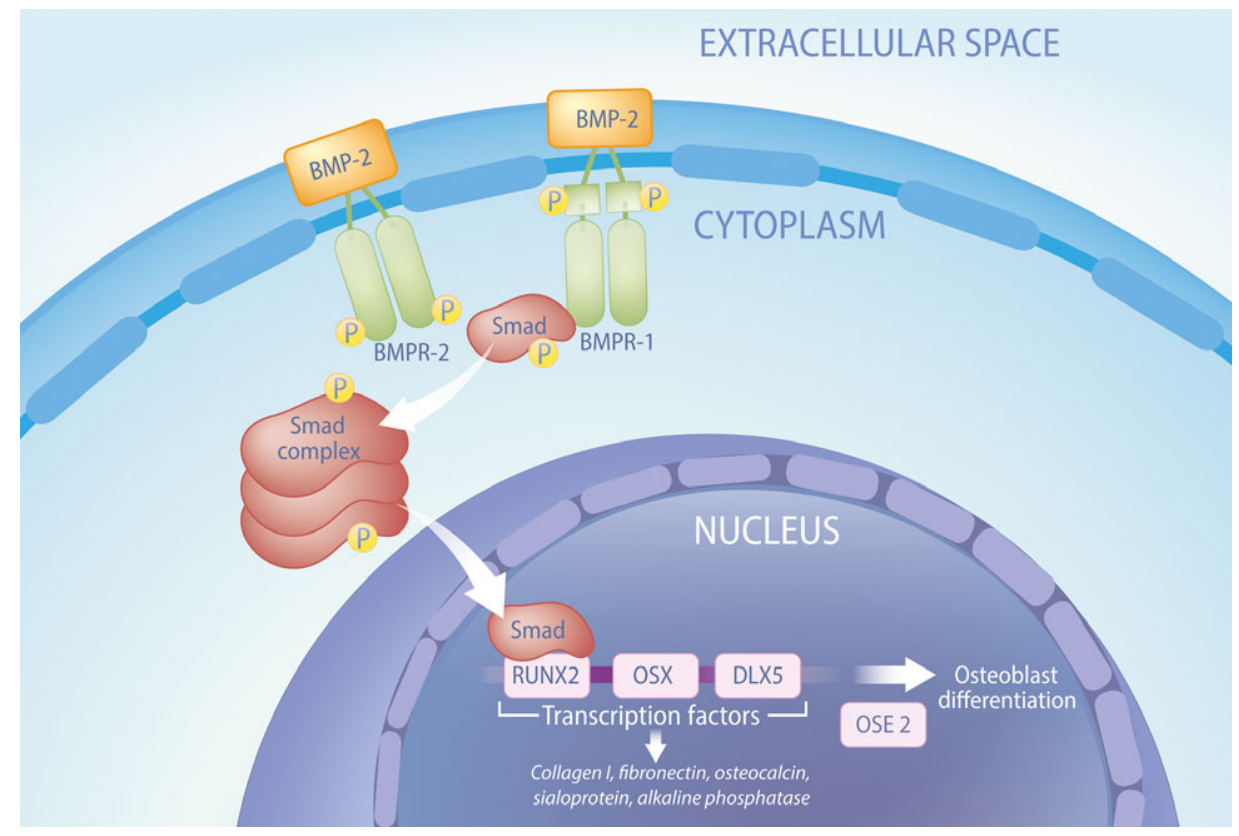

Fig. 1. Mechanism of BMP-2 and the intracellular signaling pathway. Smad: Small mothers against decapentaplegic; BMPR: BMP receptor; RUNX2: runt-related transcription factor 2; OSX: osterix; DLX5: distal-less homeobox 5 gene; OSE2: osteoblast-specific cis-acting element 2; P: phosphorylated. 


\section{Search strategy}

A search of 4 electronic databases, including PubMed/MEDLINE, EMBASE, Web of Science, and Cochrane Central, for relevant studies published in the English language from January 1990 until February 2015 was performed. The search terms used, where $\mathrm{mh}$ represented the $\mathrm{MeSH}$ terms and tiab represented title and/or abstract, were as follows: ("growth substances"[mh] OR "growth factor"[tiab] OR "biologic factors"[mh] OR "biologic agent"[tiab] OR "biologics"[tiab] OR "bone morphogenetic proteins"[mh] OR "BMP"[tiab] OR "rhBMP-2"[tiab]) AND ("dental implants"[mh] OR "maxillary sinus" $[\mathrm{mh}]$ OR "sinus floor augmentation"[mh] OR "maxillary sinus augmentation"[tiab] OR "sinus floor elevation"[tiab] OR "sinus augmentation"[tiab] OR "sinus elevation"[tiab] OR "sinus lift"[tiab] OR "sinus lifting"[tiab]).

A hand search was also carried out in dental and implant-related journals from January 2000 to February 2015, including Journal of Clinical Periodontology, Journal of Periodontology, Clinical Implant Dentistry and Related Research, International Journal of Oral and Maxillofacial Implants, Clinical Oral Implants Research, Implant Dentistry, International Journal of Oral and Maxillofacial Surgery, Journal of Oral and Maxillofacial Surgery, Journal of Dental Research, Journal of Prosthetic Dentistry, International Journal of Prosthodontics, Journal of Oral Implantology, and International Journal of Periodontics and Restorative Dentistry. European Journal of Oral Implantology was searched from Winter 2008 to Winter 2014 issues. Furthermore, a search in the references of included papers was conducted for publications that were not electronically identified. The search strategy was performed by one examiner (G-HL).

Studies were selected if they fulfilled the following inclusion criteria: human clinical trials, with data on comparison of sinus volumetric and/or histometric outcomes with and without the use of rhBMP-2 in sinus grafting procedures, with $\geq 10$ augmentation sites in each study group, and with a follow-up period of at least 6 months. Patient-centered outcome, if there is any, was also recorded. Reviews and case reports were excluded, but the bibliographies of these studies were screened for potential articles to be included. Potential articles were examined in full text by two reviewers (G-HL and GL), and their eligibility for this review was confirmed after discussion. The level of agreement between the reviewers regarding study inclusion was calculated using kappa statistics.

\section{Risk of bias assessment}

The criteria used to assess the quality of the selected randomized control trials (RCTs) were modified from the randomized clinical trial checklist of the Cochrane Center (Higgins \& Green 2011) and the CONSORT statement (Schulz et al. 2010), which provided guidelines for the following parameters: sequence generation, allocation concealment method, masking of the examiner, address of incomplete outcome data, and free of selective outcome reporting. The degree of bias was categorized as: low risk if all the criteria were met, moderate risk when only one criterion was missing, and high risk if two or more criteria were missing. Two reviewers (G-HL and GL) assessed all the included articles independently.

\section{Data extraction}

Data were extracted by two observers (G-HL and GL) independently from the papers that met the inclusion criteria. If any disagreement was found, another reviewer was consulted (H-LC). Demographic information was recorded for each study, including the study design, sample size, individual characteristics, numbers of augmented sites, techniques used, doses of rhBMPs-2, types of grafting materials, and follow-up period.

Additional variables, if there were any, recorded for each study were VBL gain, SR, bone density, and percentage of vital bone and residual grafting materials. If necessary, authors of the potentially qualified papers were contacted for more detailed data.

\section{Data analyses}

The primary outcome was VBL gain, with SR, bone density, and histometric parameters as the secondary outcomes. The risk ratio of SR and the pooled WMD of VBL, bone density, percentage of newly formed vital bone, and percentage of residual grafting materials were estimated using a computer program (RevMan version 5.0, The Nordic Cochrane Centre, The Cochrane Collaboration, Copenhagen, 2008). The contribution of each article was weighed. For studies with more than one treatment arm, the results from all arms were combined together. Random-effects meta-analyses of the selected studies were applied to minimize any bias caused by methodological differences among studies. Forest plots were generated to graphically represent the difference in primary and secondary outcomes for all included studies using augmented sites as the analysis unit. A $P$ value $=0.05$ was used as the level of significance. Heterogeneity was assessed with chi-square test and $I^{2}$ test, which ranges between $0 \%$ and $100 \%$ and lower values represent less heterogeneity. In addition, the funnel plots were used to assess the presence of the publication bias. The reporting of these metaanalyses adhered to the PRISMA (Preferred Reporting Items for Systematic Review and Meta-Analyses, Fig. 2) statement (Liberati et al. 2009).

\section{Results}

The screening process was shown in Fig. 2. Electronic and hand searches yielded 815 articles, of which 14 articles were selected for full-text evaluation after screening their titles and abstracts. Eight articles (Boyne et al. 1997; Serra et al. 2006; Tarnow et al. 2010; Jensen et al. 2012, 2013, 2014; Jensen \& Adams 2014; Luiz et al. 2014) were further excluded; the reasons for exclusion were listed in Table 1. Six articles (Boyne et al. 2005; Triplett et al. 2009; Kao et al. 2012; Froum et al. 2013b, 2014; Kim et al. 2014) were included in this systematic review. The main features and conclusions of the included studies were summarized in Table 2. An additional study (Kim et al. 2015) was identified during the revision of the current review. The data of this study were listed in Table 2 but not pooled in the meta-analyses due to disqualification of selection process.

The kappa value for inter-reviewer agreement for potentially relevant articles was 0.93 (titles and abstracts) and 1 (full-text articles), indicating an "almost perfect" agreement between the two reviewers (Landis \& Koch 1977).

\section{Features of the included studies}

Study design and patient features

Six RCTs (Boyne et al. 2005; Triplett et al. 2009; Kao et al. 2012; Froum et al. 2013b, 2014; Kim et al. 2014) were included. Most studies reported the age range and the gender of the study participants except for two studies (Froum et al. 2013b, 2014). In addition, all

\section{Table 1. Summary of the excluded articles}

\begin{tabular}{cl}
\hline Reason for exclusion & Authors/Year \\
\hline $\begin{array}{c}\text { No data reported } \\
\text { for analysis }\end{array}$ & Serra et al. (2006) \\
$\begin{array}{cl}\text { Not randomized } \\
\text { clinical trials }\end{array}$ & Boyne et al. (1997) \\
& Tarnow et al. (2010) \\
& Jensen et al. (2012) \\
& Jensen et al. (2013) \\
& Jensen \& Adams (2014) \\
& Jensen et al. (2014) \\
& Luiz et al. (2014)
\end{tabular}




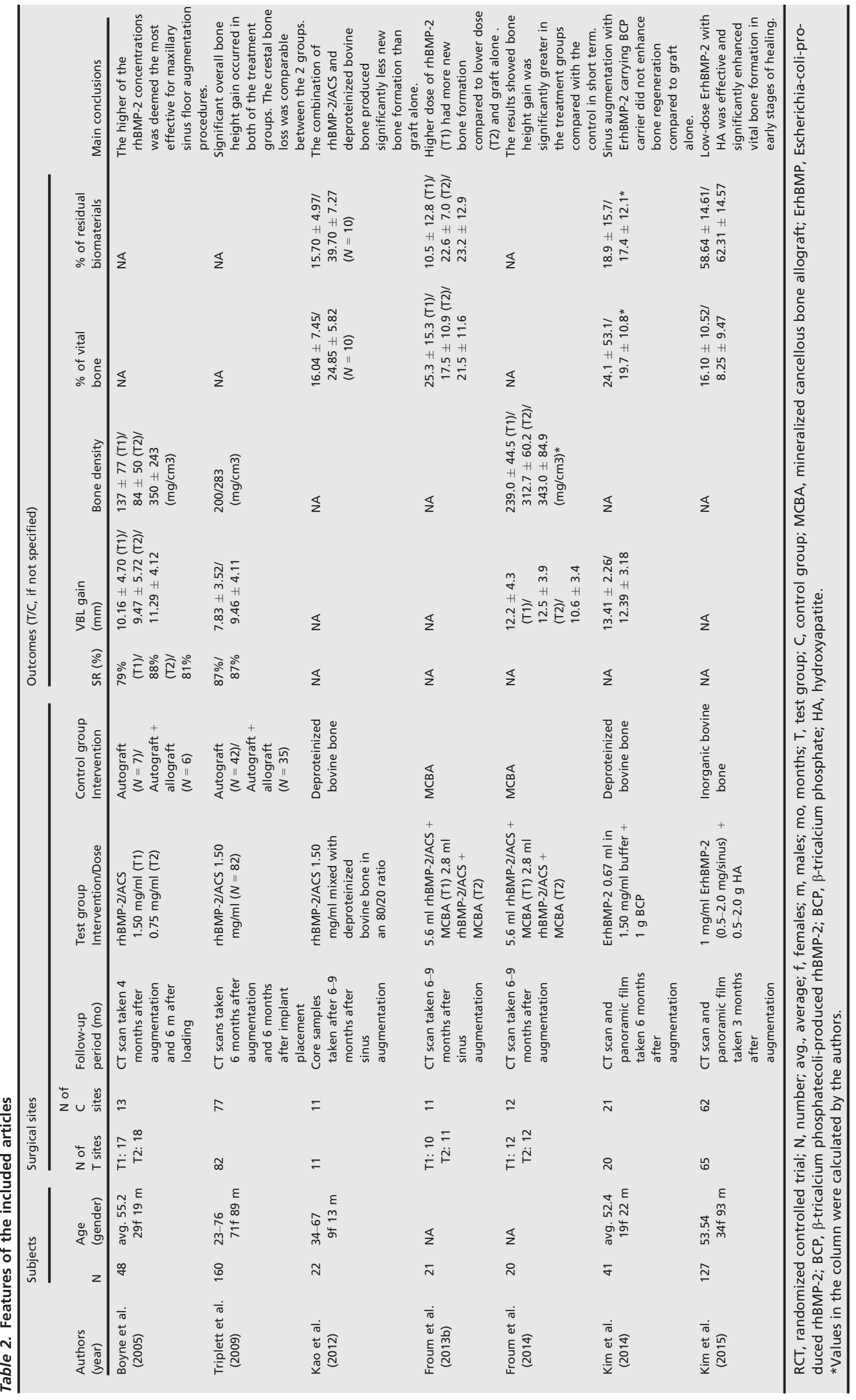


studies performed computed tomography (CT) scan within 4- to 9-month period after sinus floor augmentation to evaluate the volumetric changes. Two included studies (Boyne et al. 2005; Triplett et al. 2009) reported the SR of the implants; other studies did not report this information.

Dose of rhBMP-2 and types of grafting materials Two different types of rhBMP-2 were introduced. Five studies (Boyne et al. 2005; Triplett et al. 2009; Kao et al. 2012; Froum et al. 2013b, 2014) used rhBMP-2 derived from mammalian cells, and one study (Kim et al. 2014) used Escherichia coli-produced rhBMP2 (ErhBMP-2). Regarding the grafting materials, two studies (Boyne et al. 2005; Triplett et al. 2009) introduced rhBMP-2 with ACS (rhBMP-2/ACS) to augment maxillary sinuses as experimental group and autogenous graft in combination with allogenous graft as control group. Another two (Froum et al. 2013b, 2014) studies used rhBMP-2/ACS mixed with mineralized cancellous bone allograft (MCBA) as test group and MCBA alone as control. One study (Kao et al. 2012) used rhBMP-2/ACS mixed with deproteinized bovine bone as test group and deproteinized bovine bone alone as control group. Another one study (Kim et al. 2014) used hydroxyapatite and beta-tricalcium phosphate (BCP) at a ratio of $30: 70$ as the carrier of ErhBMP-2 solution in test group and BCP alone as control group. All the studies used rhBMP-2 of $1.50 \mathrm{mg} / \mathrm{ml}$ concentration for sinus floor augmentation; however, three studies (Boyne et al. 2005; Froum et al. 2013b, 2014) introduced an additional experimental arm to test the efficacy of $0.75 \mathrm{mg} / \mathrm{ml}$ concentration.

\section{Risk of bias assessment}

The results of risk of bias assessment for included RCTs were summarized in Table 3. Three studies were considered to have a moderate risk of bias and another 3 studies were considered to have a high risk of bias.
The results of funnel plots were reported as Supplementary Figs S1-S5 for evaluating the potential publication bias. However, the funnel plots should be interpreted with caution because the mixture of various study designs as well as limited number of studies included.

\section{Results of the meta-analyses for VBL gain}

Four studies (Boyne et al. 2005; Triplett et al. 2009; Kao et al. 2012; Froum et al. 2014) reported the data on VBL gain. The weighted mean bone gain was $10.17 \pm 0.54 \mathrm{~mm}$ in rhBMP-2 group and $10.50 \pm 0.68 \mathrm{~mm}$ in control group. The WMD of $\mathrm{VBL}$ gain was $-0.14 \mathrm{~mm} \quad(95 \% \quad \mathrm{CI}=-1.91-1.62 \mathrm{~mm}$, $P=0.87$, Fig. 3). No statistically significant difference was detected between groups. A high degree of heterogeneity $\left(I^{2}=70 \%\right.$ and $P$ value for chi-square test was 0.02) among selected studies was noticed.

\section{Results of SR}

Of the included studies, only two studies reported SR. One study (Boyne et al. 2005) reported $79 \%, 88 \%$, and $81 \%$ implant SR at grafted sites with the use of $1.5 \mathrm{mg} / \mathrm{ml}$ rhBMP-2, $0.75 \mathrm{mg} / \mathrm{ml}$ rhBMP-2, and without the use of rhBMP-2, respectively. Another study (Triplett et al. 2009) reported $87 \%$ SR for both test and control groups after 6 months of implant placement. Metaanalysis for the comparison of SR among selected studies presented an overall risk ratio of $1.00(95 \% \mathrm{CI}=0.94-1.07)$, and no statistical significance $(P=0.94)$ was found (Fig. 4) between groups treated with rhBMP-2 and treated with conventional approach. The comparisons presented a low $(P$ value for chisquare test $=0.59$ and $I^{2}$ test $=0 \%$ ) degree of heterogeneity between two studies.

\section{Results of the meta-analyses for bone density}

Three studies (Boyne et al. 2005; Triplett et al. 2009; Froum et al. 2014) reported data on bone density after sinus floor augmentation by measuring the CT scan images.
Two studies (Boyne et al. 2005; Triplett et al. 2009) reported the newly formed bone density using mineral density unit at 46 month after augmentation. However, one (Triplett et al. 2009) of the studies did not report the standard deviation of the reported data, resulting in the exclusion of this study when meta-analyzing. Another study (Froum et al. 2014) reported the bone density in Hounsfield units based on CT scans taken at 6- to 9-month follow-up. The Hounsfield units were subsequently calibrated and reported as mineral density in the current study based on an equation proposed from a previous study (Schileo et al. 2008). The WMD of bone density was $-142.42 \mathrm{mg} / \mathrm{cm}^{3} \quad(95 \% \quad \mathrm{CI}=-310.62-$ $25.78 \mathrm{mg} / \mathrm{cm}^{3}, \quad P=0.10$, Fig. 5). Although no statistically significant difference was detected between groups, a trend of favoring control group with higher newly formed bone density was noted. A high degree of heterogeneity $\left(I^{2}=82 \%\right.$ and $P$ value for chisquare test was 0.02 ) between selected studies was noticed.

\section{Results of the meta-analyses for the histometric outcomes}

Three studies (Kao et al. 2012; Froum et al. 2013b; Kim et al. 2014) reported the data on percentages of vital bone and residual grafting materials based on the examination of the core samples, retrieved 6-9 months after sinus floor augmentation. Interestingly, among these three studies, one study used allograft (MCBA), another one used xenograft (deproteinized bovine bone), and the other one used alloplastic material (BCP) as the carriers for rhBMP-2. The weighted mean percentage of vital bone was $18.21 \pm 3.56 \%$ in rhBMP-2 group and $22.69 \pm 2.63 \%$ in control group. The WMD of the percentage of vital bone was $-4.59 \%(95 \% \mathrm{CI}=-11.73-2.56 \%$, $P=0.21$, Fig. 6). No statistically significant difference was detected between groups. A moderate degree of heterogeneity $\left(I^{2}=37 \%\right.$ and $P$ value for chi-square test was 0.20 )

Table 3. Risk assessment of publication bias for the included RCTs

\begin{tabular}{|c|c|c|c|c|c|c|}
\hline Criteria (Higgins \& Green 2011) & $\begin{array}{l}\text { Boyne et al. } \\
(2005)\end{array}$ & $\begin{array}{l}\text { Triplett et al. } \\
(2009)\end{array}$ & $\begin{array}{l}\text { Kao et al. } \\
(2012)\end{array}$ & $\begin{array}{l}\text { Froum et al. } \\
(2013 b)\end{array}$ & $\begin{array}{l}\text { Froum et al. } \\
(2014)\end{array}$ & $\begin{array}{l}\text { Kim et al. } \\
(2014)\end{array}$ \\
\hline Sequence generation & Yes & Yes & Yes & Yes & Yes & Yes \\
\hline Randomization methods & $\mathrm{RCT}$ & $\mathrm{RCT}$ & $\mathrm{RCT}$ & $\mathrm{RCT}$ & $\mathrm{RCT}$ & $\mathrm{RCT}$ \\
\hline Allocation concealment method & Yes & Yes & Yes & Yes & Yes & Yes \\
\hline Examiner masked & Yes & $?$ & $?$ & Yes & Yes & No \\
\hline All patients accounted for at end of study & No & No & No & No & No & No \\
\hline Incomplete outcome data adequately addressed & Yes & Yes & NA & NA & NA & NA \\
\hline Free of suggestion of selective outcome reporting & Yes & Yes & Yes & Yes & Yes & Yes \\
\hline Estimated potential risk of bias & Moderate & High & High & Moderate & Moderate & High \\
\hline
\end{tabular}

NA, Not applicable; ?, Not reported. 


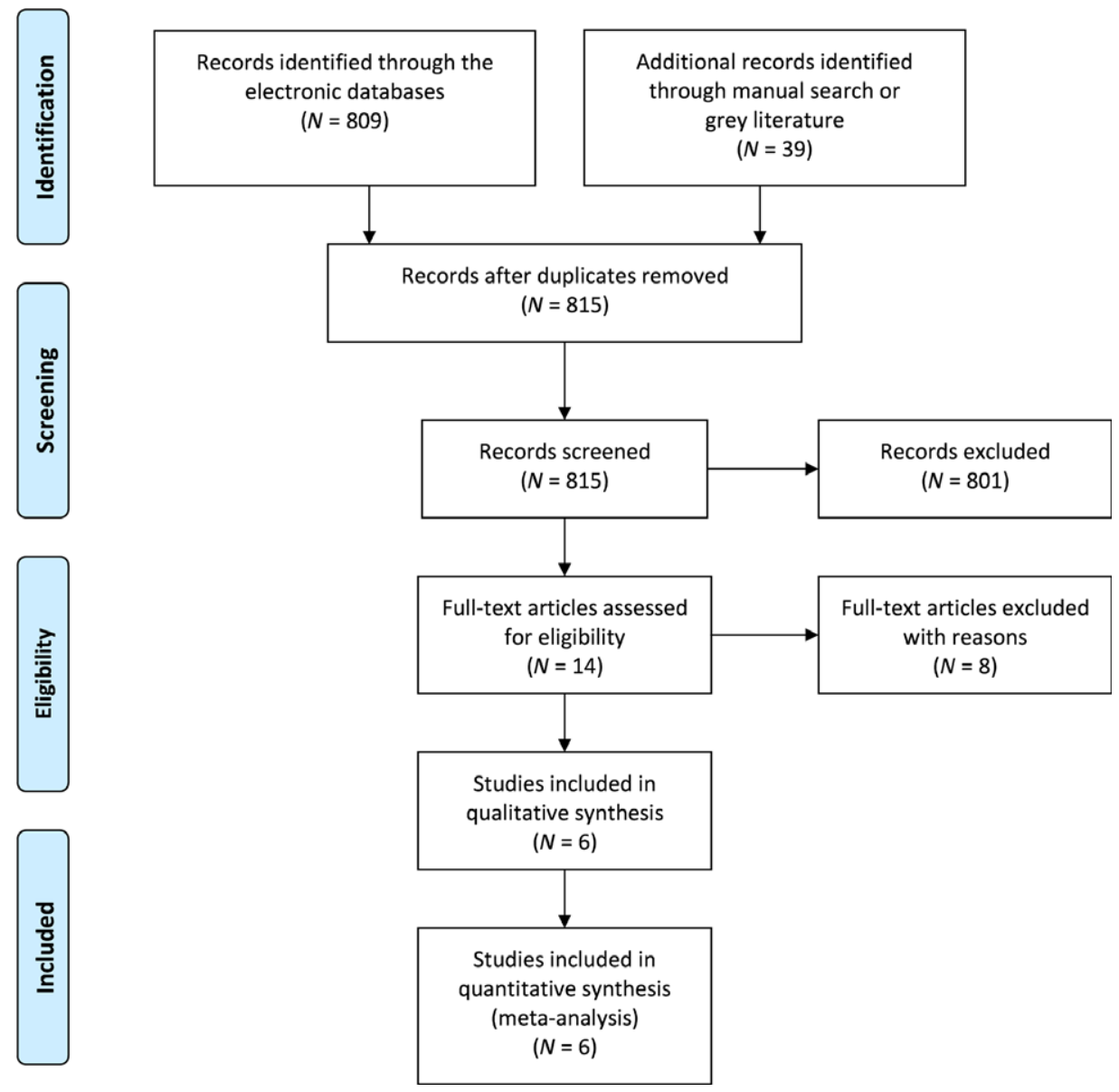

Fig. 2. PRISMA flow chart illustrates the publication selection process.

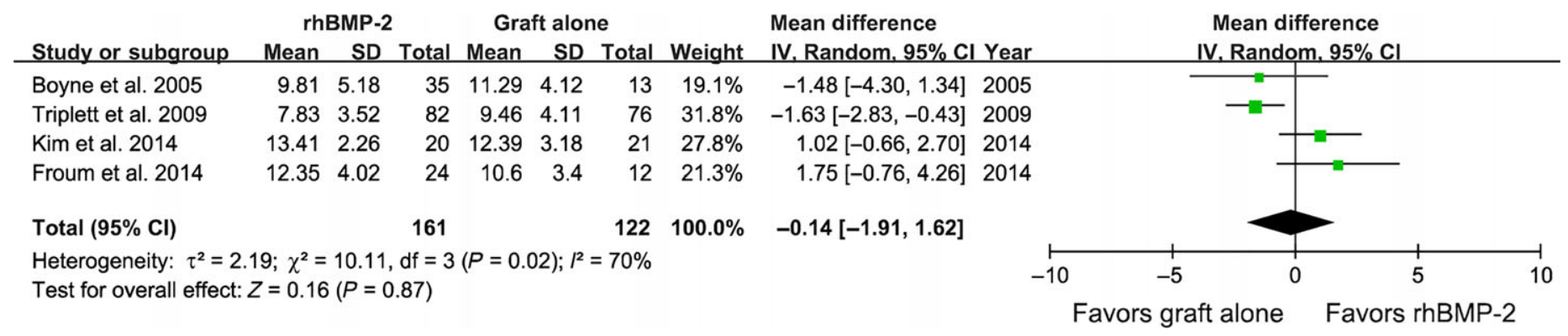

Fig. 3. Meta-analysis for the comparison of VBL gain among selected studies. The WMD of VBL gain was $-0.14 \mathrm{~mm}(95 \% \mathrm{CI}=-1.91-1.62 \mathrm{~mm}, P=0.87)$. No statistically significant difference was detected between groups. A high degree of heterogeneity $\left(I^{2}=70 \%\right.$ and $P$ value for chi-square test was 0.02$)$ among selected studies was noticed.

rhBMP-2/ACS Graft alone Risk ratio

\begin{tabular}{|c|c|c|c|c|c|c|}
\hline Study or subgroup & Events & Total & Events & Total & Weight & IV. Random, $95 \% \mathrm{CI}$ \\
\hline Boyne et al. 2005 & 131 & 156 & 51 & 63 & $19.7 \%$ & $1.04[0.90,1.19]$ \\
\hline Triplett et al. 2009 & 209 & 241 & 219 & 251 & $80.3 \%$ & $0.99[0.93,1.06]$ \\
\hline Total $(95 \% \mathrm{Cl})$ & & 397 & & 314 & $100.0 \%$ & $1.00[0.94,1.07]$ \\
\hline Total events & 340 & & 270 & & & \\
\hline
\end{tabular}

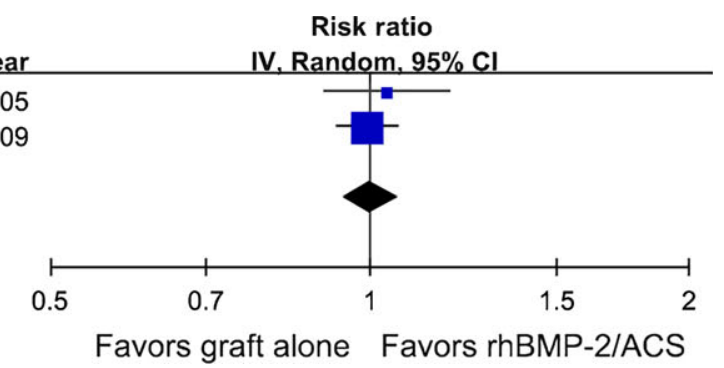

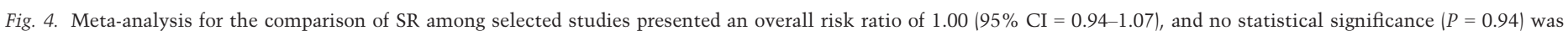
found. The comparisons presented a low ( $P$ value for chi-square test $=0.59$ and $I^{2}$ test $\left.=0 \%\right)$ degree of heterogeneity between two studies. 


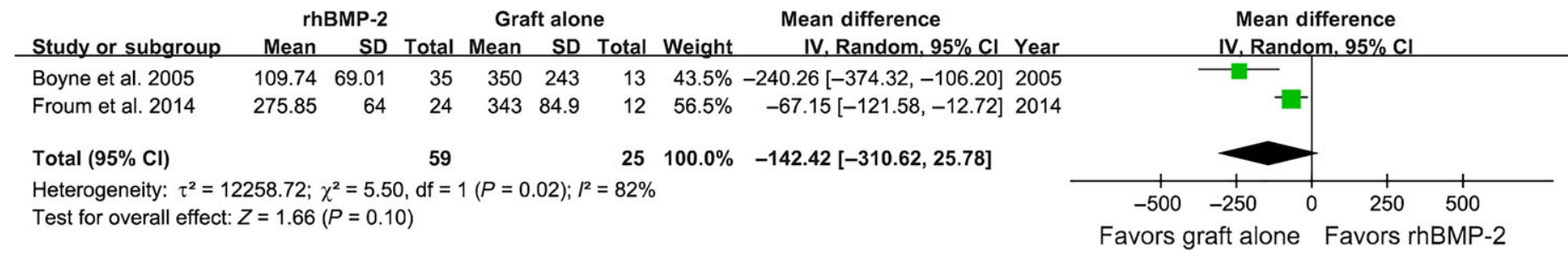

Fig. 5. Meta-analysis for the comparison of bone density among selected studies. The WMD of bone density was $-142.42 \mathrm{mg} / \mathrm{cm}^{3}\left(95 \% \mathrm{CI}=-310.62-25.78 \mathrm{mg} / \mathrm{cm}^{3}, P=0.10\right)$. Although no statistically significant difference was detected between groups, a trend of favoring control group with higher newly formed bone density was noted. A high degree of heterogeneity $\left(I^{2}=82 \%\right.$ and $P$ value for chi-square test was 0.02$)$ between selected studies was noticed.

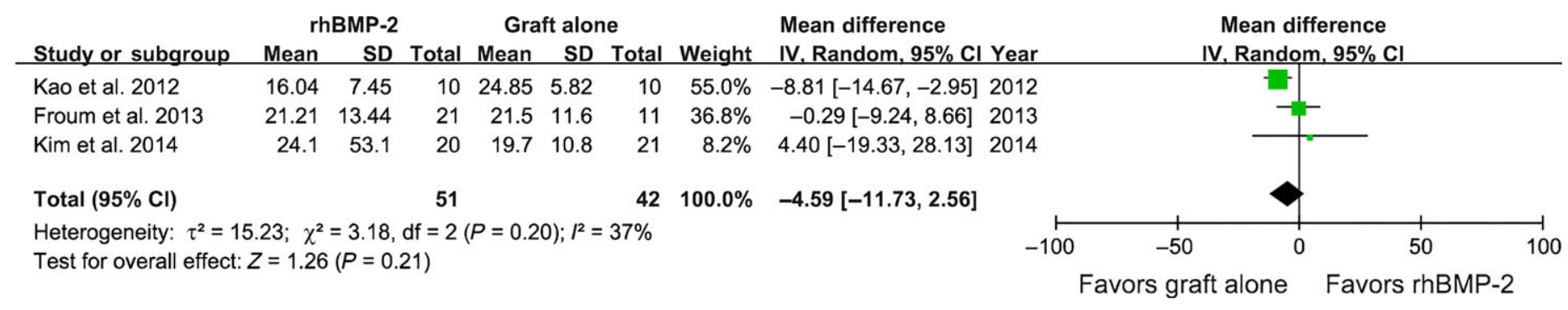

Fig. 6. Meta-analysis for the comparison of the percentage of vital bone formation among selected studies. The WMD of the percentage of vital bone was $-4.59 \%(95 \%$ $\mathrm{CI}=-11.73-2.56 \%, P=0.21)$. No statistically significant difference was detected between groups. A moderate degree of heterogeneity $\left(I^{2}=37 \%\right.$ and $P$ value for chi-square test was 0.20 ) among selected studies was noticed.

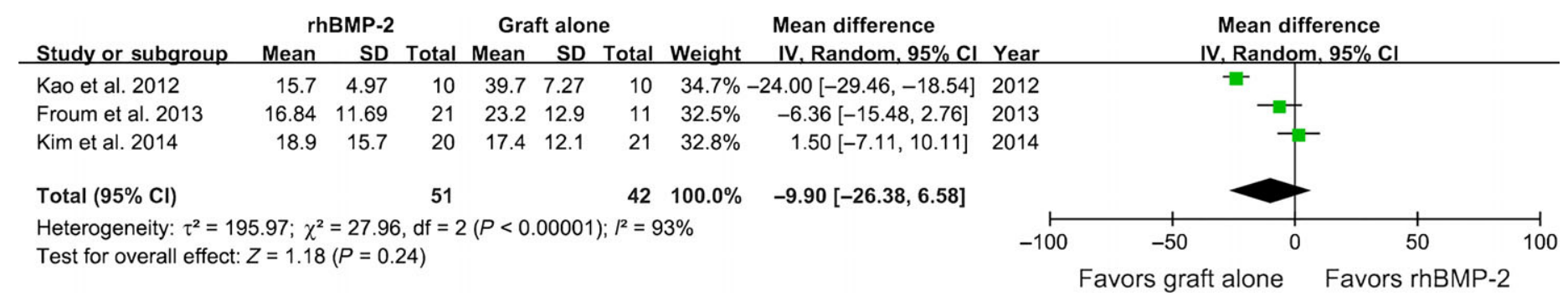

Fig. 7. Meta-analysis for the comparison of the percentage of residual bone graft particles among selected studies. The WMD of the percentage of residual bone grafting materials was $-9.90 \%(95 \% \mathrm{CI}=-26.38-6.58 \%, P=0.21)$. No statistically significant difference was detected between groups. A high degree of heterogeneity $\left(I^{2}=93 \%\right.$ and $P$ value for chi-square test was $<0.0001$ ) among selected studies was detected.

among selected studies was noticed. Similarly, the WMD of the percentage of residual bone grafting materials was $-9.90 \% \quad(95 \%$ $\mathrm{CI}=-26.38-6.58 \%, P=0.21$, Fig. 7). No statistically significant difference was detected between groups. A high degree of heterogeneity $\left(I^{2}=93 \%\right.$ and $P$ value for chi-square test was $<0.0001)$ among selected studies was presented.

Interestingly, a multicenter study (Kim et al. 2015) reporting histometric outcomes was identified during the revision of the current review. However, even the data of this article were pooled, no statistically significant difference could be detected for percentages of vital bone (WMD $=0.32 \%$ with $95 \%$ $\mathrm{CI}=-9.99-10.63 \%, \quad P=0.95)$ and residual grafting materials $(\mathrm{WMD}=-8.35 \%$ with 95\% CI $=-20.38-3.69 \%, P=0.17)$ between groups (Figures not shown).
Analysis based on the results of heterogeneity test

Of all the investigated variables, the metaanalyses presented moderate-to-high degree of heterogeneity among the studies except for SR. Several confounding factors, including various interventions, types of rhBMP-2, potential risks of bias, and follow-up period, might contribute to the moderate-to-high degree of heterogeneity. The heterogeneity could also result from combining the data of two treatment arms $(1.50 \mathrm{mg} / \mathrm{ml}$ rhBMP-2 and $0.75 \mathrm{mg} / \mathrm{ml} \mathrm{rhBMP-2)}$ to represent the outcomes of the test group in 3 studies (Boyne et al. 2005; Froum et al. 2013b, 2014). As a result, the authors have performed the analyses to compare the data with the use of $1.50 \mathrm{mg} / \mathrm{ml}$ rhBMP-2 only to the control group (data with the use of $0.75 \mathrm{mg} / \mathrm{ml}$ were not pooled), but all the comparisons still did not reach statistically significant difference between test and control groups. In addition, the same degree of the heterogeneity (low, moderate, or high) was found among the selected studies for any of the investigated variables.

When moderate-to-high degree of heterogeneity is present, a subgroup analysis or regression analysis is usually the recommended statistic method; however, these analyses were precluded in the current review due to the limited number of the included studies. Therefore, the results of the meta-analyses should be interpreted with caution.

\section{Discussion}

The results of current review demonstrated that the use of rhBMP-2 in human maxillary 
sinus floor augmentation did not show significant difference on dimensional nor histometric outcomes after 6-9 months of healing period in comparison with conventional surgical procedures. Similar results were previously reported in a systematic review published by de Freitas et al. (2015). In the study, the authors reported sinus floor augmentation following autogenous bone graft was significantly greater than the rhBMP-2/ ACS group in terms of VBL gain. Even though the present review included more randomized controlled trials, similar results were found regarding the dimensional change between groups. In addition, the current review evaluated the amount of vital bone formed after 6-9 months of the sinus floor augmentation procedure by analyzing previously published histomorphometric data. No significant difference was detected regarding the percentages of vital bone formation and residual bone grafting materials. This implies that in maxillary sinus floor augmentation, rhBMP-2 achieved similar histometric outcomes when compared to conventional sinus grafting procedure. However, it is worth noting this finding is only based on 3 studies (Kao et al. 2012; Froum et al. 2013b; Kim et al. 2014) and with only 6- to 9-month follow-up. Among these three studies, one study used MCBA (Froum et al. 2013b), another one used xenograft (Kao et al. 2012), and the other one used BCP (Kim et al. 2014) as the carriers for rhBMP-2. More interestingly, although meta-analysis did not detect any significant difference in percentage of vital bone formation, Kao et al. reported that new bone formation was compromised when a deproteinized bovine bone was used as a carrier.

Sinus floor augmentation with the use of rhBMP-2 has been widely investigated in animal studies (Nevins et al. 1996; Hanisch et al. 1997) and was subsequently introduced in human studies. In 1997, Boyne et al. (1997) published the first article using rhBMP-2/ACS in human maxillary sinus grafting procedures and reported a gain of $8.51 \mathrm{~mm}$ at 4-month follow-up. This result may appear as optimal and clinically applicable; however, after examining the data closely, the bone height gain actually ranged from $2.28 \mathrm{~mm}$ to $15.73 \mathrm{~mm}$, and only $45 \%$ of the participants met the ideal criteria for dental implant placement. On the contrary, the histological evidence had identified moderate to large number of osteoblasts and capillaries present in the newly induced bone, which suggesting the benefits of introducing rhBMP-2 to the sinus procedures given its osteoinductive property. Perhaps, the true benefit of using rhBMP-2 in this procedure might not reflect on directly gaining bone volume, but rather in the new bone induction potential. Several clinical studies (Moon et al. 2011; Riben \& Thor 2012) had shown that with a good ability of space maintenance, the optimal volumetric gain could be achieved even with the absence of bone grafting materials or biologic agents. Thus, clinicians should not expect significant gain of bone height when applying rhBMP-2 in sinus procedures; in contrast, a stable maintenance of the elevated space is the determinant for gaining vertical bone height.

Regarding the implant SR after using rhBMP-2 in sinus floor augmentation procedures, only 2 articles (Boyne et al. 2005; Triplett et al. 2009) were pooled and the metaanalysis did not detect a difference between the experimental and control groups (overall risk ratio of $1.00,95 \% \mathrm{CI}=0.94-1.07$, $P=0.94)$. Interestingly, both studies reported that the majority of implant failures occurred before prosthetic loading and resulted from inadequate bone quality during the osseointegration phase. Therefore, a possible longer healing period ( $>6$ months) might be necessary before implant placement to achieve better bone quality. Future clinical trials should be performed to determine the ideal healing time and surgical protocol when using rhBMP-2/ACS to augment sinus floor.

Cone beam CT scans have been commonly used to determine bone width and height of the alveolar ridge. Although it is not very frequent, these scans have also been used to measure bone density (Aranyarachkul et al. 2005). In the current study, meta-analysis failed to detect difference between treatment and control groups in regard of regenerated bone density after 6 months of healing; however, there was a tendency that control group demonstrated better bone density than the rhBMP-2 group. Interestingly, one study (Triplett et al. 2009) reported a significant higher bone density in the bone graft group compared with the rhBMP-2/ACS treatment group at 6 months postoperatively. However, the induced bone density in the rhBMP-2/ ACS group was significantly higher than the bone graft group at 6 months after functional loading. The authors concluded that the bone density around implants after functional loading in augmented sinus with rhBMP-2/ ACS performed as well as that of the bone graft group. Furthermore, it should be noted that a higher density of mineralized tissue shown in CT scan/radiograph at early stage of healing does not necessarily equate to higher amount of mature bone because the radiopaque/mineral property of the grafting materials might contribute to overestimation of bone quality. In two studies (Boyne et al. 2005; Triplett et al. 2009), the experimental group had no mineralized materials grafted into the sinus; another study (Froum et al. 2014) grafted a relatively smaller amount of allograft into the sinus in the treatment group while a larger amount of allograft used for the control. Therefore, special precaution should be taken when interpreting the results of this parameter.

Compared to the bone density measurement, the histomorphometric analysis of the core specimens provides more detailed information regarding the percentage of newly formed vital bone as well as residual bone particles. In a previous study, Kao et al. (2012) reported negative effect on bone formation when using rhBMP-2 in combination with deproteinized bovine bone in maxillary sinus floor augmentation. They speculated that the upregulation of receptor activator of nuclear factor kappa-B ligand might be responsible for this phenomenon. However, the current review failed to detect the difference between the treatment and control groups in terms of histometric comparisons. These outcomes were in accordance with several recent studies (Froum et al. 2013b; Kim et al. 2014). Interestingly, recent human study (Kim et al. 2015) and animal trials (Ono et al. 2014; Yon et al. 2015) had shown promising results of significant bone formation and enhanced osteointegration with the use of BMP-2. This inconsistency between human and animal studies could be explained with the early release and influence of the BMP-2 on bone formation. As most of the human studies retrieved the core specimens at the time of the implant placement (6-9 months after sinus floor augmentation), meanwhile, the bone grafting materials used in the control groups could potentially already turn over into native bone, resulting in histologically similar patterns with the treatment specimens. Yon et al. (2015) had shown that BMP-2 was released from the carrier over the first 3 days in vitro and maintained at a reduced level through day 21 and significantly enhanced local bone formation. Kim et al. (2015) also reported low-dose ErhBMP-2 significantly enhanced vital bone formation in early stages ( 3 months) of healing. Therefore, the effect of the use of rhBMP-2 in sinus floor augmentation on vital bone formation might be attained at the very early stage of the healing period, but this difference 
possibly will diminish after $6-9$ months postoperation.

Two of the included articles (Froum et al. 2013b; Kim et al. 2014) reported the difference of percentage of vital bone formation between the sites with perforated and nonperforated sinus membranes. Froum et al. $(2013 \mathrm{~b})$ reported that more bone formation $(22.37 \%)$ in perforated sinuses was associated with higher dosage of rhBMP-2/ACS. In contrast, Kim et al. (2014) showed less bone formation in perforated sinuses with the treatment of BMPs. The authors speculated that the perforated Schneiderian membrane might compromise the osteoinductive capacity of rhBMP-2. One published clinical trial (Froum et al. 2013a) demonstrated that perforated membranes did not appear to be an adverse complication in terms of vital bone formation or implant survival if properly repaired. However, rhBMP-2 was not introduced in that particular study thus the conclusion might not be applicable. The effect of membrane perforation on vital bone formation in maxillary sinus floor augmentation with the use of rhBMP-2 remains unclear at this time.

Although the current study did not show a significant difference between the rhBMP2 group and control group, there is a lack of human clinical trials to investigate potential indications, such as limited residual bone height or long span grafted area, of the use of rhBMP-2 in sinus floor augmentation procedures. The residual bone height between bone crest and sinus floor has been associated with long-term implant success and prognosis (Pjetursson et al. 2009; Del Fabbro et al. 2012). A previous study concluded that a more favorable prognosis for sinus floor augmentation procedure was noted when residual ridge height was at least $5 \mathrm{~mm}$ (Del Fabbro et al. 2012). In addition, recent studies also showed that a higher implant SR was associated with a higher initial bone height (Pjetursson et al. 2009; Soardi et al. 2013). In this case, a use of biologic agents such as BMP-2 might potentially enhance positive surgical outcomes due to its angiogenesis and osteogenesis characteristics. More human clinical trials are encouraged to investigate this clinically relevant topic.

Serious complications such as swelling or infection might occur after the use of rhBMP-2 in oral and maxillofacial procedures. Minor complications have been also reported, including post-operation bleeding and pain (Kim et al. 2014). In 2012, Woo (2012) reported that local edema, erythema, and pain were the most frequent post-operation complications after the use of rhBMP-2. In the present review, two studies (Boyne et al. 2005; Triplett et al. 2009/ reported the use of autogenous bone had a significantly greater incidence of oral edema, ecchymosis, pain, arthralgia, abnormal gait, sinusitis, skin rash, and erythema than the rhBMP-2/ ACS group, indicating the morbidity associated with bone graft harvesting. However, higher percentage $(82 \%)$ of the patients in rhBMP-2/ACS group experienced facial edema than autograft group $138 \%$, Boyne et al. 2005). The post-operation facial edema might result from an influx of fluid and cells into the treatment site (Triplett et al. 2009) during the initial phase of the wound healing. Anti-inflammatory medications could be prescribed to subside these symptoms (Tan et al. 2013).

Although the cost-effectiveness of BMP use in spinal fusion procedures has been reported (Ackerman et al. 2002; Cahill et al. 2009), this topic has not yet been widely discussed in dental field. Based on the results of the current study, an additional benefit with the use of rhBMP-2 in sinus floor augmentation procedures could not be warranted. However, rhBMP-2 might be used as an alternative to autogenous or allogenous graft. Therefore, clinicians should weigh potential benefits and downsides of applying this biologic agent in their patients. Future studies need to be conducted to combine analysis of patientcentered outcomes, procedure morbidity, and time for graft harvest as factors that may have an influence on assessing cost-effectiveness.

Several limitations of the current review are presented. First, the number of the included papers is low $(N=6)$. Second, there are various degrees of heterogeneity and publication bias. Heterogeneity is related to the presence of confounding factors within and among the selected studies, for example, different study designs, follow-up periods, types and concentrations of rhBMP-2, and grafting materials used. Although the current study clustered rhBMP-2 as a single intervention, there might be potential difference and effect when different formulations/concentrations of rhBMP-2 are used. Furthermore, most of the analyses presented a moderate to large heterogeneity among included studies; there- fore, cautious interpretation of the data is needed. Third, the study results might be influenced by the potential confounding factors, such as residual bone height and patients' health history. However, none of the included study adjusted for the related confounding factors. Fourth, volumetric changes and bone density were measured in 3-dimension, and different settings and brands of CT scan machine might have an influence on the reported outcomes. Fifth, owing to the limited comparable data, patient-centered outcomes were not statistically analyzed in the current review. Sixth, current review only included studies written in English, which could introduce a selection bias.

\section{Conclusions}

This systematic review revealed that the use of rhBMP-2 in maxillary sinus floor augmentation achieved similar clinical and histometric outcomes when compared to conventional sinus grafting procedures after a healing period of 6-9 months. However, previous studies showed the morbidity and other patientreported outcomes were improved in rhBMP2 approaches as compared to bone autograft procedures (both intraoral and extraoral bone harvesting due to donor site morbidity). Long-term studies are required to determine the cost-benefit of sinus floor augmentation procedures for patients requiring implant reconstruction.

Acknowledgements: The authors do not have any financial interests, either directly or indirectly, in the products or information listed in the article. This article was partially supported by the University of Michigan Periodontal Graduate Student Research Fund.

\section{Conflict of interest and source of funding}

The authors do not have any financial interests, either directly or indirectly, in the products or information listed in the article. This article was partially supported by the University of Michigan Periodontal Graduate Student Research Fund. 


\section{References}

Ackerman, S.J., Mafilios, M.S. \& Polly, D.W. Jr (2002) Economic evaluation of bone morphogenetic protein versus autogenous iliac crest bone graft in single-level anterior lumbar fusion: an evidence-based modeling approach. Spine 27 S94-S99.

Aranyarachkul, P., Caruso, J., Gantes, B., Schulz, E., Riggs, M., Dus, I., Yamada, J.M. \& Crigger, M. (2005) Bone density assessments of dental implant sites: 2. Quantitative cone-beam computerized tomography. The International Journal of Oral @) Maxillofacial Implants 20: 416-424.

Bowers, G., Felton, F., Middleton, C., Glynn, D., Sharp, S., Mellonig, J., Corio, R., Emerson, J., Park, S., Suzuki, J., Ma, S., Romberg, E. \& Reddi, A.H. (1991) Histologic comparison of regeneration in human intrabony defects when osteogenin is combined with demineralized freeze-dried bone allograft and with purified bovine collagen. Journal of Periodontology 62: 690-702.

Boyne, P.J., Lilly, L.C., Marx, R.E., Moy, P.K., Nevins, M., Spagnoli, D.B. \& Triplett, R.G. (2005) De novo bone induction by recombinant human bone morphogenetic protein-2 (rhBMP-2) in maxillary sinus floor augmentation. Journal of Oral and Maxillofacial Surgery 63: 1693 1707

Boyne, P.J., Marx, R.E., Nevins, M., Triplett, G., Lazaro, E., Lilly, L.C., Alder, M. \& Nummikoski, P. (1997) A feasibility study evaluating rhBMP-2/ absorbable collagen sponge for maxillary sinus floor augmentation. The International Journal of Periodontics \&f Restorative Dentistry 17: 11-25.

Cahill, K.S., Chi, J.H., Day, A. \& Claus, E.B. (2009) Prevalence, complications, and hospital charges associated with use of bone-morphogenetic proteins in spinal fusion procedures. The Journal of the American Medical Association 302: 58-66.

Carreira, A.C., Lojudice, F.H., Halcsik, E., Navarro, R.D., Sogayar, M.C. \& Granjeiro, J.M. (2014) Bone morphogenetic proteins: facts, challenges, and future perspectives. Journal of Dental Research 93: 335-345.

Chen, D., Zhao, M. \& Mundy, G.R. (2004) Bone morphogenetic proteins. Growth Factors 22: 233-241.

Chiapasco, M., Casentini, P. \& Zaniboni, M. (2009) Bone augmentation procedures in implant dentistry. The International Journal of Oral e Maxillofacial Implants 24(Suppl): 237-259.

Dai, K.R., Xu, X.L., Tang, T.T., Zhu, Z.A., Yu, C.F., Lou, J.R. \& Zhang, X.L. (2005) Repairing of goat tibial bone defects with BMP-2 gene-modified tissue-engineered bone. Calcified Tissue International 77: 55-61.

Del Fabbro, M., Corbella, S., Weinstein, T., Ceresoli, V. \& Taschieri, S. (2012) Implant survival rates after osteotome-mediated maxillary sinus augmentation: a systematic review. Clinical Implant Dentistry and Related Research 14 e159-e168.

Esposito, M., Grusovin, M.G., Felice, P., Karatzopoulos, G., Worthington, H.V. \& Coulthard, P. (2009) Interventions for replacing missing teeth: horizontal and vertical bone augmentation techniques for dental implant treatment. The
Cochrane Database of Systematic Reviews 7: CD003607.

Even, J., Eskander, M. \& Kang, J. (2012) Bone morphogenetic protein in spine surgery: current and future uses. Journal of the American Academy of Orthopaedic Surgeons 20: 547-552.

de Freitas, R.M., Spin-Neto, R., Junior, E.M., Pereira, L.A., Wikesjo, U.M. \& Susin, C. (2015) Alveolar ridge and maxillary sinus augmentation using rhBMP-2: a systematic review. Clinical Implant Dentistry and Related Research $\mathbf{1 7}$ |Suppl 1): e192-e201.

Froum, S.J., Khouly, I., Favero, G. \& Cho, S.C. (2013a) Effect of maxillary sinus membrane perforation on vital bone formation and implant survival: a retrospective study. Journal of Periodontology 84: 1094-1099.

Froum, S.J., Wallace, S., Cho, S.C., Khouly, I., Rosenberg, E., Corby, P., Froum, S., Bromage, T., Schoor, R., Norman, R. \& Tarnow, D.P. (2013b) Histomorphometric comparison of different concentrations of recombinant human bone morphogenetic protein with allogeneic bone compared to the use of $100 \%$ mineralized cancellous bone allograft in maxillary sinus grafting. The International Journal of Periodontics \& Restorative Dentistry 33: 721-730.

Froum, S.J., Wallace, S., Cho, S.C., Khouly, I., Rosenberg, E., Corby, P., Froum, S., Mascarenhas, P. \& Tarnow, D.P. (2014) Radiographic comparison of different concentrations of recombinant human bone morphogenetic protein with allogenic bone compared with the use of $100 \%$ mineralized cancellous bone allograft in maxillary sinus grafting. The International Journal of Periodontics $\Theta$ Restorative Dentistry 34: 611-620.

Govender, S., Csimma, C., Genant, H.K., ValentinOpran, A., Amit, Y., Arbel, R., Aro, H., Atar, D., Bishay, M., Borner, M.G., Chiron, P., Choong, P., Cinats, J., Courtenay, B., Feibel, R., Geulette, B., Gravel, C., Haas, N., Raschke, M., Hammacher, E., van der Velde, D., Hardy, P., Holt, M., Josten, C., Ketterl, R.L., Lindeque, B., Lob, G., Mathevon, H., McCoy, G., Marsh, D., Miller, R., Munting, E., Oevre, S., Nordsletten, L., Patel, A., Pohl, A., Rennie, W., Reynders, P., Rommens, P.M., Rondia, J., Rossouw, W.C., Daneel, P.J., Ruff, S., Ruter, A., Santavirta, S., Schildhauer, T.A., Gekle, C., Schnettler, R., Segal, D., Seiler, H., Snowdowne, R.B., Stapert, J., Taglang, G., Verdonk, R., Vogels, L., Weckbach, A., Wentzensen, A., Wisniewski, T. \& BMP-2 Evaluation in Surgery for Tibial Trauma (BESTT) Study Group (2002) Recombinant human bone morphogenetic protein-2 for treatment of open tibial fractures: a prospective, controlled, randomized study of four hundred and fifty patients. Journal of Bone and Joint Surgery 84-A: 2123-2134.

Hanisch, O., Tatakis, D.N., Rohrer, M.D., Wohrle, P.S., Wozney, J.M. \& Wikesjo, U.M. (1997) Bone formation and osseointegration stimulated by rhBMP-2 following subantral augmentation procedures in nonhuman primates. The International Journal of Oral $\leftrightarrow$ Maxillofacial Implants 12: 785-792.
Hassan, M.Q., Tare, R.S., Lee, S.H., Mandeville, M., Morasso, M., Javed, A., Wijnen, A.V., Stein, J.L., Stein, G.S. \& Lian, J.B. (2006) BMP-2 commitment to the osteogenic lineage involves activation of Runx2 by DLX3 and a homeodomain transcriptional n/etwork. Journal of Biological Chemistry 281: 40515-40526.

Higgins, J.P.T. \& Green, S. (2011) Cochrane Handbook for Systematic Reviews of Interventions. Version 5.1.0 (updated March 2011). The Cochrane Collaboration. Available at: http://www.cochranehandbook.org. Accessed March 19th, 2015.

Jensen, O.T. \& Adams, M.W. (2014) Anterior sinus grafts for angled implant placement for severe maxillary atrophy as an alternative to zygomatic implants for full arch fixed restoration: technique and report of 5 cases. Journal of Oral and Maxillofacial Surgery 72: 1268-1280.

Jensen, O.T., Adams, M., Cottam, J.R. \& Ringeman, J. (2013) Occult peri-implant oroantral fistulae: posterior maxillary peri-implantitis/sinusitis of zygomatic or dental implant origin. Treatment and prevention with bone morphogenetic protein2/absorbable collagen sponge sinus grafting. The International Journal of Oral \& Maxillofacial Implants 28: e512-e520.

Jensen, O.T., Cottam, J., Ringeman, J. \& Adams, M. (2012) Trans-sinus dental implants, bone morphogenetic protein 2 , and immediate function for allon-4 treatment of severe maxillary atrophy. Journal of Oral and Maxillofacial Surgery 70: 141-148.

Jensen, O.T., Kuhlke, K.L., Leopardi, A., Adams, M.W. \& Ringeman, J.L. (2014) BMP-2/ACS/Allograft for combined maxillary alveolar split/sinus floor grafting with and without simultaneous dental implant placement: report of 21 implants placed into 7 alveolar split sites followed for up to 3 years. The International Journal of Oral et Maxillofacial Implants 29: e81-e94.

Kao, D.W., Kubota, A., Nevins, M. \& Fiorellini, J.P. (2012) The negative effect of combining rhBMP-2 and Bio-Oss on bone formation for maxillary sinus augmentation. The International Journal of Periodontics $\leftrightarrow$ t) Restorative Dentistry 32: 61-67.

Kim, H.J., Chung, J.H., Shin, S.Y., Shin, S.I., Kye, S.B., Kim, N.K., Kwon, T.G., Paeng, J.Y., Kim, J.W., Oh, O.H., Kook, M.S., Yang, H.J. \& Hwang, S.J. (2015) Efficacy of rhBMP-2/hydroxyapatite on sinus floor augmentation: a multicenter, randomized controlled clinical trial. Journal of Dental Research 94: 158S-165S.

Kim, M.S., Lee, J.S., Shin, H.K., Kim, J.S., Yun, J.H. \& Cho, K.S. (2014) Prospective randomized, controlled trial of sinus grafting using escherichiacoli-produced rhBMP-2 with a biphasic calcium phosphate carrier compared to deproteinized bovine bone. Clinical Oral Implants Research 26: 1361-1368.

Komori, T., Yagi, H., Nomura, S., Yamaguchi, A., Sasaki, K., Deguchi, K., Shimizu, Y., Bronson, R.T., Gao, Y.-H., Inada, M., Sato, M., Okamoto, R., Kitamura, Y., Yoshiki, S. \& Kishimoto, T. (1997) Targeted disruption of Cbfa1 results in a complete lack of bone formation owing to maturational arrest of osteoblasts. Cell 89: 755-764. 
Landis, J.R. \& Koch, G.G. (1977) The measurement of observer agreement for categorical data. Biometrics 33: 159-174.

Lee, J., Susin, C., Rodriguez, N.A., de Stefano, J., Prasad, H.S., Buxton, A.N. \& Wikesjo, U.M. (2013) Sinus augmentation using rhBMP-2/ACS in a mini-pig model: relative efficacy of autogenous fresh particulate iliac bone grafts. Clinical Oral Implants Research 24: 497-504.

Liberati, A., Altman, D.G., Tetzlaff, J., Mulrow, C., Gotzsche, P.C., Ioannidis, J.P., Clarke, M., Devereaux, P.J., Kleijnen, J. \& Moher, D. (2009) The prisma statement for reporting systematic reviews and meta-analyses of studies that evaluate health care interventions: explanation and elaboration. Annals of Internal Medicine 151: W65-W94.

Luiz, J., Padovan, L.E. \& Claudino, M. (2014) Recombinant human bone morphogenetic protein 2 in augmentation procedures: case reports. The International Journal of Oral $\leftrightarrow$ Maxillofacial Implants 29: 1198-1203.

Moon, J.W., Sohn, D.S., Heo, J.U., Shin, H.I. \& Jung, J.K. (2011) New bone formation in the maxillary sinus using peripheral venous blood alone. Journal of Oral and Maxillofacial Surgery 69: 2357-2367.

Nevins, M., Kirker-Head, C., Nevins, M., Wozney, J.A., Palmer, R. \& Graham, D. (1996) Bone formation in the goat maxillary sinus induced by absorbable collagen sponge implants impregnated with recombinant human bone morphogenetic protein-2. The International Journal of Periodontics $\Theta$ Restorative Dentistry 16: 8-19.

Ono, M., Sonoyama, W., Yamamoto, K., Oida, Y., Akiyama, K., Shinkawa, S., Nakajima, R., Pham, H.T., Hara, E.S. \& Kuboki, T. (2014) Efficient bone formation in a swine socket lift model using escherichia coli-derived recombinant human bone morphogenetic protein-2 adsorbed in beta-tricalcium phosphate. Cells Tissues Organs 199: 249255.

Pjetursson, B.E., Rast, C., Brägger, U., Schmidlin, K., Zwahlen, M. \& Lang, N.P. (2009) Maxillary sinus floor elevation using the (transalveolar) osteotome technique with or without grafting material. Part I: implant survival and patients' perception. Clinical Oral Implants Research 20: 667-676.

Riben, C. \& Thor, A. (2012) The maxillary sinus membrane elevation procedure: augmentation of bone around dental implants without grafts-a review of a surgical technique. International Journal of Dentistry 2012: 105483.
Schileo, E., Dall'ara, E., Taddei, F., Malandrino, A., Schotkamp, T., Baleani, M. \& Viceconti, M. (2008) An accurate estimation of bone density improves the accuracy of subject-specific finite element models. Journal of Biomechanics 41: 2483-2491.

Schulz, K.F., Altman, D.G., Moher, D. \& Group C. (2010) Consort 2010 statement: updated guidelines for reporting parallel group randomized trials. Annals of Internal Medicine 152: 726-732.

Serra, E.S.F.M., Ricardo de Albergaria-Barbosa, J. \& Mazzonetto, R. (2006) Clinical evaluation of association of bovine organic osseous matrix and bovine bone morphogenetic protein versus autogenous bone graft in sinus floor augmentation. Journal of Oral and Maxillofacial Surgery 64: 931-935.

Sharan, A. \& Madjar, D. (2008) Maxillary sinus pneumatization following extractions: a radiographic study. The International Journal of Oral (4) Maxillofacial Implants 23: 48-56.

Sigurdsson, T.J., Fu, E., Tatakis, D.N., Rohrer, M.D. \& Wikesjo, U.M. (1997) Bone morphogenetic protein-2 for peri-implant bone regeneration and osseointegration. Clinical Oral Implants Research 8: 367-374.

Soardi, E., Cosci, F., Checchi, V., Pellegrino, G., Bozzoli, P. \& Felice, P. (2013) Radiographic analysis of a transalveolar sinus-lift technique: a multipractice retrospective study with a mean follow-up of 5 years. Journal of Periodontology 84: 1039-1047.

Sporn, M.B. \& Vilcek, J.T. (1996) The inauguration of cytokine \& growth factor reviews. Cytokine $\Theta$ Growth Factor Reviews 7: 1.

Stavropoulos, A., Becker, J., Capsius, B., Açil, Y., Wagner, W. \& Terheyden, H. (2011) Histological evaluation of maxillary sinus floor augmentation with recombinant human growth and differentiation factor-5-coated $\beta$-tricalcium phosphate: results of a multicenter randomized clinical trial. Journal of Clinical Periodontology 38: 966-974.

Tan, Y., Montgomery, S.R., Aghdasi, B.G., Inoue, H., Kaner, T., Tian, H., Terrell, R., Zhang, X., Wang, J.C. \& Daubs, M.D. (2013) The effect of corticosteroid administration on soft-tissue inflammation associated with rhBMP-2 use in a rodent model of inflammation. Spine 38: 806-813.

Tarnow, D.P., Wallace, S.S., Testori, T., Froum, S.J., Motroni, A. \& Prasad, H.S. (2010) Maxillary sinus augmentation using recombinant bone morphogenetic protein-2/acellular collagen sponge in combination with a mineralized bone replacement graft: a report of three cases. The International Journal of Periodontics \& Restorative Dentistry 30: 139-149.

Triplett, R.G., Nevins, M., Marx, R.E., Spagnoli, D.B., Oates, T.W., Moy, P.K. \& Boyne, P.J. (2009) Pivotal, randomized, parallel evaluation of recombinant human bone morphogenetic protein-2/ absorbable collagen sponge and autogenous bone graft for maxillary sinus floor augmentation. Journal of Oral and Maxillofacial Surgery 67: 19471960.

Urist, M.R. (1965) Bone: formation by autoinduction. Science 150: 893-899.

Wada, K., Niimi, A., Watanabe, K., Sawai, T. \& Ueda, M. (2001) Maxillary sinus floor augmentation in rabbits: a comparative histologic-histomorphometric study between rhBMP-2 and autogenous bone. The International Journal of Periodontics e Restorative Dentistry 21: 252263.

Woo, E.J. (2012) Adverse events reported after the use of recombinant human bone morphogenetic protein 2. Journal of Oral and Maxillofacial Surgery 70: 765-767.

Yon, J., Lee, J.S., Lim, H.C., Kim, M.S., Hong, J.Y., Choi, S.H. \& Jung, U.W. (2015) Pre-clinical evaluation of the osteogenic potential of bone morphogenetic protein-2 loaded onto a particulate porcine bone biomaterial. Journal of Clinical Periodontology 42: 81-88.

\section{Supporting Information}

Additional Supporting Information may be found in the online version of this article:

Figure S1. Funnel plot of meta-analysis of VBL gain among selected studies.

Figure S2. Funnel plot of meta-analysis of SR among selected studies.

Figure S3. Funnel plot of meta-analysis of bone density among selected studies.

Figure S4. Funnel plot of meta-analysis of percentage of vital bone formation among selected studies.

Figure S5. Funnel plot of meta-analysis of percentage of residual grafting materials among selected studies. 